\title{
Les changements sémantiques et les ambiguïtés au niveau du discours : l'exemple du fr. on
}

\author{
Winter-Froemel, Esme \\ Université de Tübingen \\ esme.winter-froemel@uni-tuebingen.de
}

\section{Introduction}

La grammaticalisation du fr. on à partir du lat. homo représente un changement bien connu dans l'histoire du français. Toutefois, son évolution ultérieure - le changement sémantique qui part de son sens indéfini et qui va vers son utilisation en tant que pronom de la première personne du pluriel ${ }^{1}$ dans le français parlé - n'a été étudié qu'à un moindre degré. Dans cette contribution, nous allons analyser dans le détail ce changement à partir de recherches de corpus (Frantext) en nous concentrant sur le stade de l'innovation, c'est-à-dire en analysant les emplois précoces du pronom dans son sens novateur (1Pl).

En d'autres termes, nos recherches auront pour but de dégager des scénarios possibles d'innovations qui initient le changement sémantique que nous venons de décrire. Ainsi, dans la partie 2, nous discuterons les emplois du pronom à partir du $\mathrm{XVI}^{\mathrm{e}}$ siècle dans lesquels le pronom sert déjà à désigner la $1 \mathrm{Pl}$, et nous opposerons deux scénarios pragmatiques bien distincts : d'une part, on peut observer des emplois qui permettent une réanalyse sémantique du pronom de la part du destinataire de l'énoncé, et d'autre part, on trouve des emplois « indirects » au sens de la 1Pl (actes de langage indirects) par l'énonciateur.

Ensuite, pour exprimer la caractéristique commune fondamentale de ces innovations possibles, nous mettrons en avant le concept de l'ambiguité et celui des contextes de transition / bridging contexts (partie 3, cf. Evans \& Wilkins 2000 : 549-550, Heine 2002 : 85-86, Marchello-Nizia 2006 : 253-264 ; Benveniste 1966 : 290). Cette dernière notion peut s'appliquer à des contextes où le sens ancien et le sens nouveau du mot peuvent coexister sans que cela gêne la communication, et on peut donc assumer que l'on a affaire ici à une situation d'ambiguïté discursive. Cette dernière notion s'oppose à un autre type d'ambiguïté, qui a été mis au centre des analyses traditionnelles des phénomènes d'ambiguité, et qui est l'ambiguïté au niveau du système linguistique.

L'opposition entre les deux types d'ambiguïté clarifiée, nous étudierons dans la partie 4 les implications théoriques qui en découlent et qui se posent pour la modélisation des changements linguistiques dans leur globalité. Nous passerons en revue les stades du changement linguistique pour les mettre en rapport avec les deux types d'ambiguité, et nous montrerons que pour expliquer des changements linguistiques tels que celui du fr. on, la notion d'ambiguité dans le discours joue un rôle fondamental. La conclusion de l'article soulignera brièvement les apports potentiels d'une approche pragmatique aux phénomènes d'ambiguïté dans le but d'arriver à mieux comprendre les changements linguistiques.

\section{Analyses d'exemples de corpus}

En ce qui concerne l'évolution sémantique du fr. on, on assume généralement que ce n'est qu'au XIX ${ }^{\mathrm{e}}$ siècle que ce pronom commence à être employé comme pronom de la $1 \mathrm{Pl}$ sans avoir une valeur stylistique spéciale (cf. Grafström $1969: 295$; Ernst $1985: 69$ ). Un des premiers auteurs chez lesquels on observe ce phénomène est Balzac (cf. l'exemple (1)). Jusqu'à présent, ce type d'emploi se restreint au français parlé (cf. Söll $1980: 137$; Koch \& Oesterreicher $2011: 177){ }^{2}$

(1) *Mademoiselle *Sophy, reprit-elle, est riche, alors on va la voir ! On fait comme si l' on ne savoit rien, et elle est reçue au château, c' est-à-dire, elle l' étoit par les anciens seigneurs ! (H. de Balzac, Annette et le criminel, 1824, p. 10 / Frantext) 
Mais il faut noter que l'on relève des emplois précoces du pronom dans son sens de la 1Pl déjà bien avant. De fait, très tôt, le pronom peut désigner, en discours, pratiquement toutes les personnes animées, et ce caractère «omnipersonnel » du pronom peut s'observer dès le moyen français (Marchello-Nizia 1997 : 225 ; Moignet 1965 : 132 ; Ernst 1985 : 69). En même temps, les travaux antérieurs s'accordent sur le fait qu'il s'agit toujours, dans ces emplois précoces du pronom dans le sens de la 1P1, d'un emploi stylistique : "On remplaçant nous (ou je) a autrefois dû avoir toujours une nuance stylistique » (Grafström 1969 : 295 ; voir aussi Söll 1980 : 135 ; Ernst 1985 : 69 ; Muller 1979 ; DHLF).

En termes de stades de changements linguistiques, cela peut s'interpréter ainsi : selon les travaux antérieurs, ce n'est qu'au XIX ${ }^{\mathrm{e}}$ siècle que le nouvel emploi de on se diffuse largement au sein de la communauté linguistique et qu'il commence à devenir conventionnel (au moins, à un certain degré et dans certains contextes d'emploi ou dans certaines variétés du français).

Soulignons d'emblée que nous adopterons, dans notre étude de ce changement, une perspective spécifique, différente de l'orientation des travaux qui analysent le pronom on ' $1 \mathrm{Pl}$ ' comme un phénomène caractéristique du français parlé, et qui mettent l'accent sur le changement dans sa globalité en diachronie : dans le but de l'expliquer par les intentions communicatives des locuteurs proposant ce nouvel emploi, nous nous intéresserons essentiellement au stade de l'innovation initiant le changement. La perspective adoptée ici est donc essentiellement synchronique, mais par rapport à une étape passée dans l'histoire de la langue.

Sachant qu'il est, dans la plupart des cas, impossible de retracer des processus de changements linguistiques jusqu'au stade de l'innovation proprement dite (au sens du tout premier emploi de l'innovation au sein de la communauté linguistique entière), nous essaierons néanmoins de dégager des emplois précoces du pronom dans son nouveau sens. Ce procédé s'explique non seulement par des nécessités pratiques ou méthodologiques (le fait que les corpus ne mettent à notre disposition qu'une petite partie de l'usage linguistique d'une certaine époque donnée), mais il peut également se justifier par des réflexions théoriques. Premièrement, on peut assumer une sorte de polygénèse des innovations à l'intérieur d'une communauté linguistique (c'est-à-dire qu'il y a des innovations parallèles et indépendantes). Et deuxièmement, si on adopte une approche basée sur le principe de l'individualisme méthodologique (cf. Keller 1984 : 64 ; 1994 : 164 ; Coseriu 1958) et si on met la perspective des locuteurs au centre des analyses, le stade de l'innovation peut être redéfini (au niveau des locuteurs et de leurs emplois discursifs individuels) comme le premier emploi dans le sens novateur selon la conscience des locuteurs, c'est-à-dire que la question fondamentale est celle de savoir si le locuteur et son interlocuteur interprètent la forme comme novatrice et « inédite » au moment de son emploi dans le discours concret.

En ce qui concerne la datation du changement étudié, il semble intéressant de noter que l'on peut relever un emploi du pronom dans un sens de $1 \mathrm{Pl}$ bien antérieur au $\mathrm{XIX}^{\mathrm{e}}$ siècle, mais qui semble ne pas être caractérisé par des effets stylistiques ou pragmatiques marqués :

(2) FOUCARAL. On y va.

(Japhet et Foucaral s'en vont.)

(P. Scarron, Don Japhet d'Arménie, 1653, p. 21, I,3 / Frantext)

Ainsi, on peut assumer que le stade de l'innovation (ou des innovations) initiant le changement sémantique du pronom vers la $1 \mathrm{Pl}$ se situe bien avant le $\mathrm{XIX}^{\mathrm{e}}$ siècle. Partant de l'attestation trouvée dans Scarron, datant de la moitié du XVII ${ }^{\mathrm{e}}$ siècle, nous analyserons donc des emplois précoces du pronom dans son nouveau sens aux $\mathrm{XVI}^{\mathrm{e}}$ et $\mathrm{XVII}{ }^{\mathrm{e}}$ siècles. Les recherches ont été effectuées sur la base de Frantext, en limitant la recherche aux documents à partir de l'an 1500. Dans bien des cas, toutefois, pour interpréter la sémantique de on dans les emplois concrets, il s'est avéré nécessaire d'avoir des extraits de textes plus longs que ceux fournis par Frantext; nous avons, le cas échéant, effectué des recherches ultérieures (dans Frantext, sur Gallica ou dans des éditions complètes des textes mêmes).

Notre question fondamentale - à savoir, quels sont les scénarios de communication dans lesquels la nouvelle interprétation du pronom indéfini on peut commencer à se réaliser - requiert ainsi des analyses 
détaillées de textes relativement longs. Nous ne présenterons donc pas une vaste étude de corpus avec des tendances quantitatives ; nous avons opté par contre pour une forte orientation qualitative de nos analyses d'extraits de corpus. Dans un deuxième temps, nous avons mis au centre de nos analyses certains textes et certains types de textes qui nous ont paru particulièrement importants pour expliquer l'évolution sémantique du pronom. D'une part, nous avons analysé toutes les occurrences du fr. on dans trois comédies de Scarron, à savoir Le Jodelet ou le Maistre valet (1645), Don Japhet d'Arménie (1653) et Le Gardien de soy-mesme (1655) (au total, environ 400 occurrences). Ces textes ont l'avantage de présenter des situations de communication dialogiques où il est, dans la plupart des cas, possible de reconstituer les scénarios de communication (p.ex. les connaissances du locuteur et de l'interlocuteur, les connaissances que le locuteur attribue à son interlocuteur, la hiérarchie sociale dans la situation de communication, etc.).

D'autre part, nous avons dépouillé six textes de type religieux ${ }^{3}$, en analysant pour chaque texte 50 occurrences du pronom. Le domaine de la religion semble particulièrement important dans notre contexte, dans la mesure où il permet souvent une réinterprétation de nous dans un sens universel, se référant à la chrétienté entière et devenant par là interchangeable avec certains emplois du pronom on (cf. infra).

Comment peut-on expliquer la naissance de on comme pronom de la 1P1 ? Dans nos analyses des emplois précoces du on de la $1 \mathrm{Pl}$, notre hypothèse de départ sera que ce sont des emplois ambigus qui sont à l'origine de ce changement sémantique. De plus, selon les recherches antérieures, on peut formuler l'hypothèse que dans les emplois précoces de on avec une valeur personnelle, on observe des effets stylistiques (cf. supra). Par conséquent, on pourrait supposer que le changement commence à partir des emplois stylistiques et qu'il résulte d'un affaiblissement des effets pragmatiques / stylistiques (cf. Grafström 1969 : 295 ; Söll 1980 ; 135 ; Ernst 1985 : 69 ; Muller 1979).

Mais les travaux antérieurs avancent encore une deuxième hypothèse pour expliquer le changement. Selon cette hypothèse, ce sont certains emplois du pronom - des emplois avec une valeur générique - qui constitueraient le point point de départ pour l'évolution ultérieure vers la 1Pl (cf. Creissels, à paraître, p. 25 ; Giacalone \& Sansò 2007). Cette hypothèse repose sur une distinction entre deux types différents d'emploi du pronom à l'intérieur des emplois indéfinis (c'est-à-dire à l'intérieur des emplois traditionnels, qui représentent le sens ancien du pronom avant le changement étudié). Ces deux types sont opposés comme les lectures génériques vs. épisodiques (cf. Cabredo Hofherr 2008 : 36-37 ; Fløttum, Jonasson \& Norén 2007 : 24-31) ou comme «existential on » / on référentiel vs. " gnomic on » / on non-référentiel (cf. Creissels, à paraître) (cf. les exemples en (3) et en (4)).

(3) on générique / gnomique / non référentiel : On ne doit jamais faire cela. / On lit ces choses dans les journaux. / Si l'on ne fait pas attention, on attrape un rhume. (cf. Cabredo Hofherr 2008 : 36) / En vieillissant, on a besoin d'aide. / En Italie, on sait préparer les pâtes. (cf. Creissels, à paraître, p. 13)

(4) on épisodique / existentiel / référentiel : Hier, on m'a volé mon vélo. / A l'accueil on m’a dit que je pouvais utiliser l'ascenseur. (cf. Cabredo Hofherr 2008: 36) / On frappe à la porte. / On a retrouvé ton porte-monnaie. / On a dormi dans ce lit. (cf. Creissels, à paraître, p. 8)

Or, s'agit-il vraiment d'hypothèses contradictoires, ou peuvent-elles éventuellement se reconcilier dans une certaine mesure ? Pour répondre à cette question, passons maintenant aux analyses d'exemples de corpus.

Pour le $\mathrm{XVI}^{\mathrm{e}}$ siècle, on relève déjà certains emplois du pronom qui semblent se prêter (potentiellement) à une réanalyse sémantique (pour la notion de réanalyse sémantique cf. Detges \& Waltereit 2002). Même si on note, dans ces extraits de textes, de faibles différences sémantiques dans l'emploi de on et de nous nous réfère à un groupe déterminé de personnes (p.ex. les personnes dont on relate les expériences sur l'île en (5)), par contre, les propositions contentant on peuvent s'interpréter dans un sens plus général, se référant à un ensemble indéfini, plus abstrait de personnes (c'est-à-dire que ces emplois pourraient s'analyser comme des emplois génériques ou gnomiques / non référentiels de on ; p.ex. la phrase « de sorte qu'on ne sçavoit voir la terre » en (5) s'applique théoriquement à toute personne se rendant à cet endroit) -, les référents du pronom on incluent le groupe défini par nous, et ce dernier groupe de 
personnes représente en quelque sorte le groupe le plus immédiatement concerné par l'expérience décrite. Ainsi, une réinterprétation de on au sens de la $1 \mathrm{Pl}$ de la part de l'interlocuteur semble tout à fait possible, et il semble également possible d'expliquer le choix du pronom on par un désir de variation stylistique.

(5) Cependant que nous estions en cette isle, tous les jours nous avions une reverdie de gros vent de la terre venant de l'est, qui elevoit le sablon de sorte qu'on ne sçavoit voir la terre, et en venoit la poussiere jusques en nos navires qui nous gastoit les yeux. (P. Crignon, La Navigation de Jean et Raoul Parmentier, ca 1529, p. 17 / Frantext; italiques miennes, de même pour les exemples suivants)

Des observations analogues peuvent se faire pour les extraits donnés en (6) et en (7). L'emploi pratiquement interchangeable de nous et de on est particulièrement visible en (7), où les deux pronoms apparaissent dans la série des descriptions des actions du groupe (déterminé) de personnes : nous trouvasmes - on voyoit - on fit - nous retirasmes.

(6) Et ainsi que ledit messire *Jehan *George estoit au soupper et que l'ostesse et luy se devisoient de pluseurs choses, elle print à parler des questains et tout en riant, luy demande qu'il venoit faire en leur ville. «Car, se m'eyde *Dieu, dit elle, on n'y ait que faire de vous, car nous nous passons bien de questains. Nous avons bien, dit celle femme, autre part à mettre nostre argent. (P. de Vigneulles, Les Cent Nouvelles nouvelles, 1515, p. 174 / Frantext)

(7) En retournant vers la rive, environ quelque espace de ce lieu, nous trouvasmes Vassé tout nu, couché aux dents, percé tout au travers par les reins, si qu'on luy voyoit les entrailles, et plusieurs coups de dards au dos, aux fesses et aux costez; et fut retourné. Les tripes luy sortoit du ventre, et avoit plusieurs coups de dards au col et à la gorge. En ce lieu on fit sa fosse, en priant Dieu qu'il luy plust avoir pitié de leurs ames. Cela fait, nous retirasmes vers la fontaine qui estoit vers le nord [...] (P. Crignon, La Navigation de Jean et Raoul Parmentier, ca 1529, p. 27 / Frantext)

Pour la première occurrence du pronom on en (7) (« on voyait »), on peut assumer qu'il s'agit encore d'un emploi générique : on se réfère, en principe, à toute personne voyant le mort, mais il est clair que le groupe le plus immédiatement concerné par cette expérience est le groupe de personnes sur l'île dont fait partie le locuteur. Par là, dans le contexte donné, une réinterprétation au sens de la $1 \mathrm{Pl}$ semble tout à fait possible. Pour la deuxième occurrence (« on fit »), la situation est différente. Ici, il s'agit clairement d'un emploi épisodique ou personnel, dans lequel on désigne la $1 \mathrm{Pl}$, et on peut supposer qu'il est motivé par des raisons stratégiques: soit le locuteur veut éviter de spécifier l'agent, soit l'emploi de on peut s'expliquer par un désir de variation stylistique. Un fort argument en faveur de cette dernière hypothèse émerge d'une analyse du contexte dans lequel se situe l'extrait cité : dans les descriptions qui le précèdent immédiatement, le locuteur décrit la découverte de deux autres morts, et dans ces descriptions, on trouve de nombreux emplois de nous. Ces répétitions peuvent donc motiver le choix du pronom on pour des raisons de variation stylistique, d'autant plus que l'on trouve, quelques phrases avant, une formulation parallèle à celle en question (« on fit sa fosse »), dans laquelle le pronom nous est employé (« nous luy fismes sa fosse ») :

(8) Notre capitaine et le capitaine du Sacre y vinrent au dernier quart du jour, nos deux batteaux du Sacre esquippez de mariniers et arquebuziers, avec des futailles pour l'eau; arrivasmes au point du jour à la terre où il y a une moult belle descente, et de prime face allasmes chercher les lieux où nos gens avoient esté tuez; et trouvasmes Breant enterré hors le bois sur le sablon, enseveli en des feuilles de palme, et enfouy au sablon environ un demy pied ou trois paulmes; et dessus avoient mis une grosse boise seche et planté un roseau au bout de la fosse. Nous ostasmes un petit de sablon pour voir lequel c'estoit, et vismes à son visage que c'estoit Breant; et si aperceusmes aucuns coups qu'il avoit en la poitrine et au visage, puis fut recouvert, et entrasmes au bois pour chercher les autres; et assez avant dedans, nous trouvasmes Jacques l'Escossois tout nud, couché aux dents, ayant diverses playes par tout le corps; fut retourné et luy vismes la poitrine toute pleine de coups de dards et puoit desjà fort. Aupres du lieu où il estoit, nous luy físmes sa fosse et l'ensepulturasmes dedans. (P. Crignon, La Navigation de Jean et Raoul Parmentier, ca 1529, p. 27 
/ Frantext; Pierre Crignon: Poète et navigateur. Euvres en prose et en vers. Présentées et annotées par John Nothnagle. Summa Publications, 1990, p. 27)

En ce qui concerne les hypothèses de départ que nous avons formulées plus haut, il semble intéressant de noter que l'on peut observer ici un désir de variation stylistique, mais ces emplois ne semblent pas confirmer l'hypothèse d'une valeur stylistique spéciale de on; tout au contraire, la variation stylistique résultant de l'emploi de on à côté de nous présuppose en quelque sorte leur équivalence sémantique (dans le contexte donné).

Dans les emplois plus tardifs, les observations que nous venons de faire se confirment, c'est-à-dire que l'on trouve encore des emplois où on semble interchangeable avec nous, de sorte qu'une réinterprétation sémantique du pronom devient possible (cf. l'exemple en (9)). A cet égard, on note que la réinterprétation sémantique semble particulièrement facile dans des textes ou contextes religieux, où on et nous réfèrent à l'humanité ou à la chrétienté entière (voir l'exemple en (10), cf. aussi (11) et (12)) : ici, le contexte n'invite pas à une interprétation plus spécifique de on (comme dans les exemples précédents, où sont décrites les actions d'un groupe déterminé de personnes), mais le contexte suggère plutôt une interprétation plus globale de nous dans un sens universel, de sorte que on et nous deviennent à nouveau interchangeables.

(9) *ÉRASTE. En quel lieu sommes-nous?

De quelque part qu'on tourne, on ne voit que des fous.

(Molière, Les Fâcheux, 1662, p. 61, II,2 / Frantext)

(10) Il faut que pour rendre l'homme heureux elle [la religion, EWF] lui montre qu'il y a un Dieu, qu'on est obligé de l'aimer, que notre vraie félicité est d'être en lui et notre unique mal d'être séparé de lui, qu'elle reconnaisse que nous sommes pleins de ténèbres qui nous empêchent de le connaître et de l'aimer [...] (B. Pascal, Pensées, 1662, p. 134 / Frantext)

(11) Si cela estoit, et si les hommes ne pouvoient jamais dire la verité, nous serions tous barbares les uns aux autres. On ne s' entendroit pas mieux qu' on faisoit, quand les langues furent confonduës. (J.-L. (Guez de) Balzac, Dissertations chrestiennes et morales, 1654, p. 298 / Frantext)

(12) [...] mais que, comme les chemins vont et viennent, ou plustost (car ilz ne bougent pas) on va et vient par eux, nous allons toute nostre vie à la mort, esperants de revenir en vie pour resusciter en estat bienheureux. (Anonyme, Voyage d'Italie, 1606, p. 77 / Frantext)

De l'analyse des exemples que nous venons de présenter, nous pouvons conclure que l'on note, de fait, un certain nombre d'emplois où le pronom on, dans sa valeur générique ou gnomique, peut se réinterpréter au sens de la 1Pl, de sorte que l'on peut assumer qu'il s'agit d'emplois précoces qui pourraient avoir mis en route le changement sémantique du pronom.

Toutefois, dans des textes du XVII ${ }^{\mathrm{e}}$ siècle, on trouve aussi des contextes d'emploi ambigus qui se caractérisent de façon tout à fait différente aux niveaux sémantique et pragmatique : dans divers exemples, le pronom a clairement une valeur épisodique ou personnelle tout en étant (potentiellement) ambigu (cf. aussi l'exemple (7) analysé plus haut). Ainsi, dans l'exemple (13), on sert à définir un groupe précis et limité de personnes (Jodelet - déguisé en Dom Juan - exclus, cf. l'extrait du texte) - ceux qui connaissent l'assassin -, et nous apprenons par la suite que Dom Fernand, le locuteur qui emploie ce pronom, sait très bien lui-même qui est l'assassin. Par conséquent, on peut se réinterpréter ici dans un sens de $1 \mathrm{Sg}$ ou de $1 \mathrm{Pl}$.

(13) JODELET. [...] De venir sottement m'avertir d'un outrage

Que je ne sçavois point et ne voulois sçavoir.

DOM FERNAND. [...] Il faut sans vous servir de la valeur d'un autre

Aujourd'huy s'il se peut voir l'espée à la main

Celuy qu'on sçait avoir tué vostre germain,

Il le tua la nuit, soit hazard, soit vaillance,

Vous devez vistement en faire la vengeance. 
JODELET. Fut-ce la nuit?

DOM FERNAND. La nuit !

(P. Scarron, Le Jodelet ou le Maistre valet, 1645, p. 58-59, IV,5 / Frantext)

Dans l'extrait donné en (14), on pourrait d'abord assumer une valeur générique du pronom, " c'est ce qu'on va sçavoir » se référant à un groupe non déterminé de personnes. Toutefois, dans le contexte de la communication, il devient clair que l'on peut déterminer de manière plus précise le groupe de personnes intéressées par cette nouvelle. Ensuite, comme Honorius et Placidie comptent parmi ce groupe, une interprétation au sens de la $1 \mathrm{Pl}$ semble également possible.

(14) *HONORIUS. Tu le vas voir paroistre,

Demeure.

*PLACIDIE. Mais, Seigneur, connoissez-vous le traistre?

Pour qui conspiroit-on?

*HONORIUS. C'est ce qu'on va sçavoir

Par ceux des assassins qui sont en mon pouvoir,

Du Ciel dans leur deffaite admirez la justice.

(T. Corneille, Stilicon, 1660, p. 105, V,4 / Frantext)

Analysons encore un troisième exemple où on prend une valeur épisodique / personnelle et potentiellement ambiguë. En (15), Haman, qui est le ministre du Roi de Perse, relate d'abord les décisions prises par le Roi (le châtiment des Juifs), et leur exécution projetée (« on va mettre la main à cette grande affaire »). Dans le contexte de la communication, on peut assumer que ce choix du pronom indéfini est motivé par une stratégie de politesse ou de prudence, c'est-à-dire que Haman préfère, dans un premier temps, se «dissimuler » et ne pas spécifier qui exécutera les actions, et ce n'est que sur la demande d'Esther qu'il précise son rôle actif («c'est par mes conseils... »). Ici, le locuteur figure donc encore une fois parmi les référents de on, et une réanalyse de on au sens de la 1Pl devient possible.

(15) HAMAN. [...] Le Roi qui voit ces maux, et qui connaît leur source

Veut se montrer bon Prince en arrêtant leur course.

Ainsi par un Arrêt rendu secrètement

Il a de tous les Juifs conclu le châtiment.

ESTHER. Quand doit s'exécuter cet Arrêt salutaire?

HAMAN. On va mettre la main à cette grande affaire.

ESTHER. Mais par quelle clarté favorable à l'État

A-t-on de ces méchants découvert l'attentat?

HAMAN. C'est par mes soins, Madame, heureux et nécessaires

Que le Roi reconnaît ses secrets adversaires,

Et c'est par mes conseils que son bras irrité

Va faire choir sur eux le foudre mérité.

(P. du Ryer, Esther, 1644, p. 62, IV,2, v. 1361-1372 / Frantext ; <http://www.theatre-

classique.fr/pages/programmes/edition.php?t=../documents/ DURYER_ESTHER.xml\#S42>)

Dans d'autres exemples, l'emploi stratégique du pronom indéfini est encore plus manifeste. Si nous regardons les exemples (16) et (17), nous constatons que le pronom on est employé dans des actes de langage indirects. Dans les deux cas, le locuteur qui utilise le pronom fait une promesse (« on va vous satisfaire » / « on va vous les faire savoir »). Dans le contexte pragmatique de l'énoncé, il est clair que ces phrases permettent une implicature pragmatique qui renforce le message, de sorte que le locuteur s'autooblige à accomplir la promesse et que le pronom peut s'interpréter dans un sens de $1 \mathrm{Sg}$ ou de $1 \mathrm{Pl}$ («je vais / nous allons vous satisfaire » / « je vais / nous allons vous les faire savoir »). Toutefois, il faut noter que cette valeur de on n'a qu'un statut pragmatique, c'est-à-dire qu'elle pourrait être annulée comme c'est le cas pour toutes les implicatures pragmatiques ( «j'ai bien dit que l'on va vous satisfaire, mais je n'ai nullement dit que c'est moi qui le ferai / c'est nous qui le ferons »).

(16) *LÉLIE. Quoi ? j'obtiendrois de vous le bonheur que j'espère?

Vous pourriez...? 
*ANDRÈS. Tout à l'heure on va vous satisfaire.

(Molière, L'Étourdi ou les Contre-temps, 1663, p. 225, V,4 / Frantext)

*PHILAMINTE. [...] Holà ! Je vous ai dit en paroles bien claires, Que j'ai besoin de vous.

*HENRIETTE. Mais pour quelles affaires ?

*PHILAMINTE. Venez, on va dans peu vous les faire savoir.

(Molière, Les Femmes savantes, 1673, p. 141, III,3 / Frantext)

Pour conclure, les extraits de corpus montrent que la réinterprétation sémantique du pronom on peut avoir lieu dans divers contextes qui se caractérisent de façon tout à fait différente aux niveaux sémantique et pragmatique. Les analyses ont confirmé la possibilité d'emplois ambigus entre le on générique / gnomique et le on de la 1Pl. Dans ces cas, l'emploi du pronom indéfini par l'énonciateur ne s'explique pas par une volonté de produire des effets pragmatiques ou de suggérer des implicatures pragmatiques, mais représente un choix tout à fait neutre, qui pourrait s'expliquer par des raisons stylistiques: l'énonciateur veut éviter la répétition du pronom nous et choisit ainsi le pronom on qui, dans le contexte de son énoncé, peut s'interpréter comme faisant référence au même groupe de personnes. Ainsi, on n'a pas besoin de postuler une innovation sémantique de la part du locuteur, mais en même temps, il semble possible que le destinataire du message (ré-)interprète le pronom en tant que pronom de la 1Pl. De cette façon, de telles réanalyses induites par l'interlocuteur peuvent initier le changement linguistique en question.

Toutefois, nous avons également constaté que, à part ces emplois se prêtant à une réanalyse, on peut observer un deuxième type d'emplois ambigus, qui se basent, par contre, sur la valeur existentielle de on (emploi référentiel) ${ }^{4}$. Il s'agit là d'actes de langage indirects, par lesquels le locuteur suggère à son interlocuteur une certaine interprétation pragmatique du pronom. Plus spécifiquement, le locuteur veut donner à entendre au destinataire qu'il fait lui-même partie des personnes auxquelles le pronom réfère, de sorte qu'il s'auto-oblige à faire un acte déterminé etc. (p.ex. celui de satisfaire un désir de l'interlocuteur, cf. les exemples (16) et (17)). En même temps, cette interprétation pourrait, comme toutes les implicatures pragmatiques, être annulée, c'est-à-dire que l'énonciateur pourrait se retirer sur l'interprétation alternative, basée sur le sens lexicalisé, indéfini, du pronom (qui est le sens ancien) : p.ex. «...mais je n'ai nullement dit que c'est nous qui... ». En ce sens, pour que le changement sémantique du pronom puisse se mettre en route, le destinataire doit ratifier l'emploi pragmatique du locuteur, par lequel le nouveau sens s'introduit.

Ces deux scénarios que nous venons de décrire semblent être bien distincts. Toutefois, on peut également noter qu'ils partagent une caractéristique fondamentale : dans les deux cas, l'interprétation du pronom dans l'énoncé est ambiguë, dans la mesure où les deux interprétations (qui correspondent aux sens ancien et nouveau du mot) sont permises par le contexte de la communication. Approfondissons maintenant nos réflexions sur cette situation d'ambiguité au niveau discursif.

\section{Les contextes de transition (bridging contexts), l'ambiguïté dans le discours et l'ambiguïté dans le système de la langue}

Comme nous venons de le voir, les deux scénarios d'innovations que nous avons contrastés - les actes de langage indirects et la réanalyse - partagent une caractéristique commune fondamentale : dans les deux cas, il y a deux interprétations possibles de l'énoncé, qui sont toutes les deux compatibles avec le contexte de la communication. Cette caractéristique renvoie à un terme-clé qui a été forgé dans la linguistique récente pour analyser les changements sémantiques : les contextes de transition ou bridging contexts (Evans \& Wilkins 2000 ; cf. Heine 2002 : 85-86, Marchello-Nizia 2006 : 253-264 ; Benveniste 1966 : 290). Partant du constat - désormais largement reconnu - que les changements sémantiques impliquent un stade de polysémie, dans lequel le mot a acquis un sens nouveau, mais conserve également son sens ancien, les auteurs soulignent que pour expliquer la naissance de telles polysémies, on doit assumer des 
contextes d'emploi où le nouveau sens du mot est impliqué de façon pragmatique (mais où il n'est pas encore devenu conventionnel) :

\begin{abstract}
It has become a standard assumption that semantic change from meaning $\mathrm{A}$ to $\mathrm{B}$ normally involves a transitional phase of polysemy where a form has both meanings (Wilkins 1981, 1996, Sweetser 1990, Heine 1997:82). Less often articulated is that this phase of polysemy (what Heine calls the stage of overlap) is typically preceded by a phase where meaning B is only contextually implicated but not yet lexicalized as a distinct sense (cf. Traugott 1989). That is to say, meaning B often comes into existence because a regularly occurring context supports an inference-driven contextual enrichment of A to B. In these contexts, which we term BRIDGING CONTEXTS, speech participants do not detect any problem of different assignments of meaning to the form because both speaker and addressee interpretations of the utterance in context are functionally equivalent, even if the relative contributions of lexical content and pragmatic enrichment differ. Subsequently this contextual sense may become lexicalized to the point where it need no longer be supported by a given context. (Evans \& Wilkins 2000 : 549-550)
\end{abstract}

Ainsi, dans les bridging contexts, le sens nouveau et le sens ancien sont équivalents d'un point de vue fonctionnel, de sorte que la divergence des interprétations ne se fait pas noter dans la communication. Sans approfondir la question de savoir si cette description est valable pour tous les types de changements sémantiques ${ }^{5}$, insistons sur le fait que la caractéristique fondamentale que nous venons de mentionner renvoie directement à un autre terme-clé de la sémantique : l'ambiguïté.

On note d'emblée que différents aspects de l'ambiguïté se croisent dans la description citée plus haut. D'une part, le stade de polysémie auquel Evans et Wilkins font référence représente un stade où le mot en tant qu'entité virtuelle du répertoire des signes de la langue - est ambigu. D'autre part, l'ambiguïté qui peut s'observer dans les bridging contexts est d'une tout autre sorte, car ici, comme le soulignent les auteurs, le nouveau sens ne s'introduit qu'au niveau pragmatique et ne représente pas encore un sens lexicalisé du mot.

Les approches antérieures à l'ambiguïté se sont intéressées surtout au premier des deux types d'ambiguïté que nous venons de mentionner. Selon les définitions traditionnelles, l'ambiguïté se caractérise par une coexistence de plusieurs sens d'un mot ou d'une structure syntaxique donnés (ambiguïté lexicale et ambiguïté syntaxique, respectivement, cf. les exemples (18) et (19)). Pour les cas d'ambiguïté lexicale, on peut encore en opposer deux types principaux, qui sont les polysémies et les homonymies. Dans toutes ces manifestations de l'ambiguïté, il s'agit donc d'ambiguïtés au niveau du système linguistique (de la grammaire ou du lexique); ces ambiguïtés peuvent, bien sûr, se refléter dans des emplois discursifs concrets des éléments ambigus, mais ceci n'est pas nécessairement le cas, dans la mesure où le contexte de l'énoncé exclut souvent une des interprétations théoriquement possibles (cf. les exemples (20) et (21), où l'interprétation est orientée vers le sens de 'cambriolage' et 'l'amour éprouvé par la mère', respectivement).

(18) vol 'déplacement dans l'air', 'cambriolage'

(19) l'amour de la mère: 'l'amour éprouvé par la mère' vs. 'l'amour éprouvé envers la mère'

(20) Le crime n'aurait pas eu le vol pour mobile. (Gide, cf. PR s.v. vol $_{2}$ )

(21) [...] Elle sait le soulager, le consoler. C'est l'amour de la mère qui permet à l'enfant d'aller toujours plus loin et d'évoluer. Elle fait preuve de patience et de tolérance. $[\ldots](<\mathrm{http}: / /$ parisyoga.fr/th\%C3\%A9matiques-yoga/yoga-famille-soci $\% \mathrm{C} 3 \% \mathrm{~A} 9 \mathrm{t} \% \mathrm{C} 3 \% \mathrm{~A} 9 /$ hommage- $\% \mathrm{C} 3 \% \mathrm{~A} 0$-la$\mathrm{m} \% \mathrm{C} 3 \% \mathrm{~A} 8 \mathrm{re} />$, 04-11-2011)

Sur la base de ce dernier constat, il semble donc nécessaire de postuler un deuxième type fondamental d'ambiguïté en-dehors des ambiguïtés virtuelles en langue (au niveau du système linguistique) : les ambiguïtés effectives en discours (cf. Kerbrat-Orecchioni 2005 ; Biville 2005 : 67 ; Bauer, Knape, Koch \& Winkler 2010 ; Winter-Froemel \& Zirker 2010). Ces ambiguïtés se caractérisent par le fait qu'ici, 
l'ambiguité est un fait « réel » et directement observable, dans la mesure où un énoncé particulier se prête à deux interprétations différentes (p.ex., l'énoncé en (22) peut s'interpréter de deux façons différentes : 'les clefs de la maison sont emmenées au garage' / 'les clefs sont emmenées de la maison au garage'; de même, en (23), la cure peut se référer soit à la 'paroisse', soit à un 'traitement médical').

(22) Il emmène les clefs de la maison au garage.

(23) Le prêtre était enchanté de sa cure. (cf. Fleury $1971: 31$ )

La différence entre les deux types nous semble fondamentale, dans la mesure où les analyses des ambiguités en question concernent des phénomènes linguistiques d'un ordre tout à fait différent et requièrent d'être étudiées suivant une méthodologie foncièrement différente. Pour caractériser les deux approches fondamentales, on peut se référer à la distinction de différents niveaux d'analyse de faits linguistiques proposée par Coseriu (1958 : 25-28). Selon lui, les phénomènes linguistiques peuvent être de trois ordres fondamentaux : universaux (niveau de la faculté humaine du langage), historiques (niveau des langues particulières) et individuels (ou actuels; niveau des énoncés concrets). Les deux niveaux concernés ici sont les niveaux historique (ambiguïtés au niveau de la langue, c'est-à-dire, ambiguïtés virtuelles qui se manifestent dans un état historique d'une langue donnée) et individuel (ambiguités au niveau du discours, qui se manifestent dans un énoncé concret).

Ainsi, les ambiguïtés au niveau de la langue se situent dans le système d'une langue donnée (un état historique, synchronique de la langue) ; le constat qu'un certain mot (de la langue) est ambigu implique que l'on fait abstraction de ses usages concrets dans des énoncés particuliers pour analyser sa sémantique. Pour les ambiguïtés au niveau du discours, par contre, on a affaire à des énoncés concrets et individuels, par exemple à des attestations du mot dans des corpus. Pour analyser le fonctionnement de l'ambiguïté dans le discours, il semble fondamental d'analyser non seulement la sémantique du mot dans la langue (qui est à la base de son emploi dans le discours), mais aussi d'étudier les facteurs contextuels et pragmatiques qui influent sur l'emploi du mot et qui peuvent soit opérer vers une désambiguïsation soit évoquer de nouveaux sens pragmatiques («ambiguïsation» d'un mot jusque-là non ambigu en langue). De cette façon une reconsidération des phénomènes d'ambiguïté dans une perspective pragmatique s'avère ici, à notre avis, nécessaire.

\section{Les deux types d'ambiguïté et les changements linguistiques donnant naissance à de nouvelles ambiguïtés}

Le dernier aspect que nous venons d'évoquer renvoie à la question des changements linguistiques. Quels sont les rapports entre les deux types d'ambiguïté, et de quelle manière peut-on les associer aux différents stades des changements linguistiques?

En ce qui concerne le rapport entre les deux types d'ambiguïtés, on peut constater que les ambiguités au niveau du discours remontent souvent à une ambiguïté au niveau du système linguistique (p.ex., le mot cure en (23) est ambigu au niveau de la langue [dans le français d'aujourd'hui], de sorte que peut se manifester également une ambiguïté dans le discours si ce mot est employé, voir le cas A dans le Tableau 1).

Toutefois, l'ambiguïté du mot dans la langue n'est une condition ni nécessaire ni suffisante pour que se manifeste une ambiguïté dans le discours : premièrement, comme nous l'avons vu en (20) et (21), des mots et des structures ambigus dans la langue ne le sont souvent pas dans les discours (c'est-à-dire que les mots sont désambiguïsés dans le contexte de l'énoncé concret, voir le cas B dans le Tableau 1). Deuxièmement, considérant les changements linguistiques dans lesquels un sens nouveau s'introduit dans la langue, on doit assumer un stade (celui de l'innovation) où, au niveau du système de la langue, le mot n'a que son sens « ancien » (donc, on ne note pas d'ambiguïté au niveau de la langue), mais où s'introduit un sens nouveau dans l'énoncé (ambiguïté au niveau du discours, cf. les exemples cités dans la partie 2, ainsi que le cas $\mathrm{C}$ dans le Tableau 1). Ensuite, bien sûr, cet emploi novateur peut se diffuser au sein de la langue et devenir conventionnel, de sorte que l'ambiguïté devient part intégrante du système de la langue. 
De cette façon, de nombreux changements linguistiques représentent des cas de naissance de nouvelles ambiguïtés de mots jusque-là non ambigus, et ces ambiguïtés peuvent ensuite se refléter ou non au niveau des discours individuels (cf. les cas A et B, respectivement).

\section{NIVEAU INDIVIDUEL DU DISCOURS}

A

B

C

$$
\text { emploi ambigu }=\text { innovation }
$$

p.ex. On est obligé d'aimer Dieu.

['pronom indéfini' / 'pronom de la 1Pl']

\section{NIVEAU HISTORIQUE DE LA LANGUE (SYSTÈME)}

ambiguïté

cure 'paroisse' / 'traitement médical'

ambiguïté

vol 'déplacement dans l'air', 'cambriolage'

pas d'ambiguïté

on 'pronom indéfini'

\section{Après conventionnalisation de l'innovation :}

emplois ambigus (cf. cas A) et emplois non ambigus (cf. cas B)

p.ex. On ne sait jamais. ['pronom indéfini'] /

On part - tu viens ? ['pronom de la 1Pl']/

On verra. ['pronom indéfini' / 'pronom de la 1Pl']

Tableau 1: Ambiguïtés au niveau de la langue et ambiguïtés au niveau du discours

\section{Conclusion}

Dans cette contribution, nous avons essayé de montrer les apports potentiels d'une approche des changements sémantiques qui est orientée vers le stade des innovations et qui part de la perspective des locuteurs qui proposent les emplois novateurs. En analysant le changement du pronom on, qui acquiert une valeur de 1Pl dans l'histoire du français, nous avons décrit différents scénarios possibles d'innovations en nous intéressant aux contextes d'emploi et aux stratégies pragmatiques qui peuvent être liées à l'emploi du pronom on (scénarios de réanalyse sémantique de la part de l'interlocuteur vs. scénarios d'emplois « indirects» du pronom de la part du locuteur). Dans ces analyses, la notion de l'ambiguïté s'est révélée d'une importance fondamentale pour expliquer les changements. En même temps, il faut noter que l'ambiguïté se manifeste de manière assez différente dans les deux scénarios 
décrits : tandis que dans les réanalyses, l'ambiguïté est non intentionnelle, et n'est même pas ressentie par les interlocuteurs (l'interlocuteur introduit une interprétation novatrice qui est, toutefois, entièrement compatible avec le contexte de la communication, et la divergence des interprétations reste, dans un premier temps, inaperçue), les actes de langage indirects impliquent des emplois stratégiques de l'ambiguïté de la part du locuteur. De plus, nous avons vu qu'il faut encore distinguer deux autres aspects fondamentaux d'ambiguïté qui entrent en jeu dans des processus de changements linguistiques. Après avoir proposé une distinction entre les ambiguïtés en langue et les ambiguïtés en discours pour bien séparer ces deux aspects, nous avons, pour finir, analysé comment ces deux types d'ambiguïté se manifestent et interagissent dans différents stades de changements linguistiques. Nous avons essayé de montrer que les analyses des extraits de corpus selon des paramètres sémantiques et pragmatiques peuvent contribuer à une meilleure compréhension du changement linguistique étudié. De manière plus générale, il semble ainsi très prometteur de reconsidérer les recherches sur les phénomènes d'ambiguïté, de les reformuler dans une perspective pragmatique, orientée vers les locuteurs, et de lier cette conception de l'ambiguïté aux études des changements linguistiques.

\section{Références bibliographiques}

DHLF = Rey A. (1998). Dictionnaire historique de la langue française. 3 vols. Paris : Dictionnaires Le Robert.

Frantext $=$ Base textuelle FRANTEXT. $<$ http://www.frantext.fr $/>$.

Gallica $=$ Gallica, bibliothèque numérique. $<$ http://gallica.bnf.fr $/>$.

PR = Rey, A., Rey-Debove, J. (2000). Le nouveau Petit Robert. Dictionnaire alphabétique et analogique de la langue française. Paris : Dictionnaires Le Robert.

Bauer, M., Knape, J., Koch, P., Winkler, S. (2010). Dimensionen der Ambiguität. Zeitschrift für Literaturwissenschaft und Linguistik, 158, 7-75

Benveniste, É. (1966). Problèmes sémantiques de la reconstruction. É. Benveniste. Problèmes de linguistique générale. Paris : Gallimard, t. I, 289-307 (extrait de Word, 10, 1954, 251-264).

Biville, F. (2005). Formes et fonctions de l'ambiguïté volontaire dans les textes latins. L. Basset, F. Biville (éds.). Les jeux et les ruses de l'ambiguïté volontaire dans les textes grecs et latins. Actes de la Table Ronde organisée à la Faculté des Lettres de l'Université Lumière-Lyon 2 (23-24 novembre 2000). Lyon: Maison de l'Orient et de la Méditerranée, 57-71.

Cabredo Hofherr, P. (2008). Les pronoms impersonnels humains - syntaxe et interprétation. Modèles linguistiques, $X X I X-1$, vol. $57,35-56$.

Coseriu, E. (1958). Sincronía, diacronía e historia. El problema del cambio lingüistico. Montevideo : Universidad de la República (Reimpresión fotomecánica, Tübingen, 1969).

Creissels, D. (2008). Impersonal and related constructions : a typological approach. Lectures at the University of Tartu, June 02-03 2008, <http://www.deniscreissels.fr/index.php?pages/autres-documentst\%C3\%83\%C2\%A91\%C3\%83\%C2\%A9chargeables>, 09.11.2011.

Creissels, D. (à paraître). Impersonal pronouns and coreference : the case of French on. À paraitre dans : S. Manninen, K. Hietaam, E. Keiser, V. Vihman (éds.) : Passives and Impersonals in European Languages. $<$ http://www.deniscreissels.fr/index.php?pages/autres-documentst\%C3\%83\%C2\%A91\%C3\%83\%C2\%A9chargeables>, 09.11.2011.

Detges, U., Waltereit, R. (2002). Grammaticalization vs. Reanalysis: a Semantic-Pragmatic Account of Functional Change in Grammar. Zeitschrift für Sprachwissenschaft, 21, 151-195.

Ernst, G. (1985). Gesprochenes Französisch zu Beginn des 17. Jahrhunderts. Direkte Rede in Jean Héroards „Histoire particulière de Louis XIII“ (1605-1610). Tübingen : Niemeyer. 
Evans, N., Wilkins, D. (2000). In the Mind's Ear: Semantic Extensions of Perception Verbs in Australian Languages. Language, 76, 546-592.

Fleury, D. (1971). L’ambiguïté. Communication et langages, 9/9, 30-40.

Fløttum, K., Jonasson, K., Norén, C. (2007). ON. Pronom à facettes. Bruxelles : De Boeck.

Giacalone, A., Sansò, A. (2007). The spread and decline of indefinite man-constructions in European languages: An areal perspective. P. Ramat, E. Roma (eds.). Europe and the Mediterranean as linguistic areas: convergencies from a historical and typological perspective. Amsterdam / Philadelphia : Benjamins, 95-131.

Grafström, A. (1969). On remplaçant nous en français. Revue de linguistique romane, 33, 270-298.

Heine, B. (2002). On the Role of Context in Grammaticalization. I. Wischer, G. Diewald (eds.). New Reflections on Grammaticalization. Amsterdam / Philadelphia : Benjamins, 83-101.

Keller, R. (1984). Bemerkungen zur Theorie des sprachlichen Wandels. Zeitschrift für germanistische Linguistik, 12, 63-81.

Keller, R. ( $\left.{ }^{2} 1994\right)$. Sprachwandel. Von der unsichtbaren Hand in der Sprache. Tübingen : Francke $\left({ }^{1} 1990\right)$.

Kerbrat-Orecchioni, C. (2005). L'ambiguïté : définition, typologie. L. Basset, F. Biville (éds.). Les jeux et les ruses de l'ambiguïté volontaire dans les textes grecs et latins. Actes de la Table Ronde organisée à la Faculté des Lettres de l'Université Lumière-Lyon 2 (23-24 novembre 2000). Lyon : Maison de l'Orient et de la Méditerranée, 13-36.

Koch, P., Oesterreicher, W. (2011). Gesprochene Sprache in der Romania. Französisch, Italienisch, Spanisch. Berlin, New York : de Gruyter ( $\left.{ }^{1} 1990\right)$.

Marchello-Nizia, C. (1997). La langue française aux XIV et XVe siècles. Paris : Nathan.

Marchello-Nizia, C. (2006). Grammaticalisation et changement linguistique. Bruxelles : De Boeck-Duculot.

Moignet, G. (1965). Le pronom personnel français. Essai de psycho-systématique historique. Paris : Klincksieck.

Muller, C. (1970). Sur les emplois personnels de l'indéfini « on ». C. Muller. Langue française et linguistique quantitative. Recueil d'articles. Genève : Slatkine, 65-72.

Söll, L. (21980). Gesprochenes und geschriebenes Französisch. Berlin : Schmidt.

Winter-Froemel, E., Zirker, A. (2010). Ambiguität in der Sprecher-Hörer-Interaktion. Linguistische und literaturwissenschaftliche Perspektiven. Zeitschrift für Literaturwissenschaft und Linguistik, 158, 76-97.

\footnotetext{
${ }^{1}$ Alternativement on pourrait parler, bien sûr, de la $4{ }^{\text {ème }}$ personne (P4), terme qui soulignerait davantage que cette catégorie ne représente pas simplement un pluriel de la $1 \mathrm{Sg} / \mathrm{P} 1$ ('je'), comme le note justement un des lecteurs.

${ }^{2}$ Partant de ce constat, il serait intéressant d'approfondir les réflexions sur l'évolution du français on en se demandant s'il y a des facteurs spécifiques, liés aux conditions de communication du français parlé (immédiat communicatif), qui interviennent ici (cf. Koch \& Oesterreicher 2011). Ainsi, on pourrait avancer l'hypothèse que les emplois indirects du pronom sont plus fréquents dans des situations de communication qui impliquent un contact direct entre le locuteur et l'interlocuteur. De même, on pourrait analyser dans quelle mesure le contexte de la communication contribue, dans les situations d'immédiat communicatif, à restreindre le groupe de personnes immédiatement concernées par le pronom (cf. l'emploi de l'all. Man sieht sich, littéralement 'On se voit', pour prendre ses adieux dans des situations de communications informelles; ici, les personnes désignées par man 'on' sont clairement le locuteur et l'interlocuteur. Néanmoins, il faut noter que les emplois du pronom indéfini allemand pour désigner la 1P1
} 
sont des emplois pragmatiques, c'est-à-dire que cette interprétation du pronom n'a pas été conventionnalisée dans le système de la langue).

${ }^{3}$ F. de Sales (saint), Introduction à la vie dévote (1619); P. de Bérulle, Discours de l'estat et des grandeurs de Jésus par l'union ineffable de la divinité avec l'humanité (1623); A. Arnauld, De la Fréquente Communion où les Sentimens des Pères, des Papes et des Conciles, touchant l'usage des sacremens de Pénitence et d'Eucharistie, sont fidèlement exposez... par M. Antoine Arnauld... (1643); J.-L. (Guez de) Balzac, Dissertations chrestiennes et morales (1654) ; P. Nicole, Essais de morale contenus en divers traités, t. 2 (1671) ; B. Pascal, Pensées (1662).

Creissels souligne que dans ce type d'emploi, on a perdu sa valeur de pronom et devrait s'analyser plutôt comme un marqueur impersonnel (cf. Creissels 2008, 13).

5 Pour des changements métaphoriques, caractérisés par une relation de similarité métaphorique entre le sens ancien et le sens nouveau, une telle coexistence des interprétations semble difficilement concevable. Par exemple, pour l'expression faire le pont, il semble difficile d'imaginer des contextes où l'interprétation figurative de pont puisse coexister avec son interprétation au sens lexical du mot ('construction reliant deux points'), qui est à l'origine de la métaphore. L'hypothèse des bridging contexts semble donc se limiter à certains types de changements sémantiques, et il se pose ainsi la tâche de déterminer plus dans le détail quelles sont les relations sémantiques qui permettent un tel stade d'équivalence fonctionnelle entre le sens ancien et le sens nouveau.

${ }^{6}$ On laisse de côté ici la question des changements linguistiques dus à des homonymies dites gênantes. Celles-ci peuvent s'illustrer par le remplacement de l'a.fr. moudre 'traire' par l'a.fr. traire, qui est souvent expliqué par la volonté des locuteurs d'abolir, par leurs innovations (l'emploi de l'a.fr. traire 'tirer' pour exprimer le concept de TRAIRE), l'ambiguïté (dans la langue) du mot moudre ('broyer (des grains)' < lat. molere ; 'traire' < lat. mulgēre). Pourtant, il reste à prouver de quelle manière ce facteur peut déterminer les choix des locuteurs individuels, car, comme on l'a souvent souligné, dans les changements linguistiques, les locuteurs n'ont normalement ni la moindre intention de changer la langue ni la conscience de le faire ; par contre, leurs innovations ne s'expliquent que par des finalités immédiates dans la situation de communication donnée (cf. Coseriu 1958). 